\title{
Attentional focusing instructions and force production
}

\section{David C. Marchant*}

Department of Sport and Physical Activity, Edge Hill University, Ormskirk, Lancashire, UK

\section{Edited by:}

Nicola Jane Hodges, University of British Columbia, Canada

\section{Reviewed by:}

Nancy Mcnevin, University of Windsor, Canada

David Sherwood, University of

Colorado, USA

\section{${ }^{*}$ Correspondence:}

David C. Marchant, Department of Sport and Physical Activity, Edge Hill University, Ormskirk, Lancashire L39 4QP, UK.

e-mail: david.marchant@edgehill.ac.uk
Research progress assessing the role of attentional focusing instructions on skill acquisition and performance has lead researchers to apply this approach to force production tasks. Initial converging evidence indicates that force production tasks are sensitive to verbal instruction; externally focused instructions (onto movement outcomes, or onto the object force is being exerted against) are shown to be more beneficial than internally focused instructions (focusing attention onto the movements being executed). These benefits are observed for maximal and accurate force production, as well as the maintenance of force production in prolonged tasks. A range of mechanisms are identified supporting the proposal that an external focus promotes movement efficiency in line with energy and effort conservation. Future research is required to assess how this developing body of work interacts with the broader understanding of psychological and physiological factors implicated in the effective production, maintenance, and limitation of maximal or sub-maximal forces.

\section{Keywords: external focus instructions, internal focus instructions, muscular force, muscular force endurance,} movement efficiency
Zachry et al. (2005) proposed that attentional focusing instructions could influence maximal muscular force production or endurance tasks. This was in light of their own and the growing body of evidence demonstrating the benefits of externally focused instructions (e.g., attention is directed to an effect that a movement has on the external environment) compared to internally focused instructions (e.g., attention directed toward bodily movements involved during skill execution) on movement execution and learning. The benefits of externally focused instructions have been demonstrated in the acquisition and performance of a range of movements. Sports skills have included Golf (Wulf et al., 1999; Wulf and Su, 2007; Bell and Hardy, 2009), basketball (Al-Abood et al., 2002; Zachry et al., 2005), volleyball and soccer kicks (Wulf et al., 2002), tennis (Wulf et al., 2000), and dart throwing (e.g., Marchant et al., 2007, 2009). This is in addition to research demonstrating benefits during standing and dynamic balance (e.g., McNevin et al., 2003; Wulf, 2008). In rehabilitation settings, externally focused instruction benefited stroke patients recovery of reaching movements (Fasoli et al., 2002) and assisted Parkinson's patients balance maintenance (Landers et al., 2005; Wulf et al., 2009). These beneficial effects of an external focus have been observed in comparison to both internal instruction and control (no attentional instruction) conditions (e.g., McNevin and Wulf, 2002; Wulf and McNevin, 2003; Wulf et al., 2003; Landers et al., 2005). When no specific instruction is provided in control conditions, this research also demonstrates that participants appear to direct their attention toward the control of movements resulting in deteriorated performance similar to that in internal focus condition. For reviews of this body of work, see Wulf (2007).

Externally focused instructions have been associated with a range of mechanisms, purported to demonstrate the use of automatic and less attentionally demanding control processes to produce movements more efficiently. These include: reduced probe reaction times during a dynamic balance task (Wulf et al., 2001a); increased accuracy and reduced electromyographic (EMG) activity during basketball free-throw shooting (Zachry et al., 2005) and dart throwing (Lohse et al., 2010a); and higher frequency movement characteristics during balance (e.g., Wulf et al., 2000, 2001b; McNevin et al., 2003). An external focus has also been shown to result in lower EMG activity compared to internal focus and control conditions using force generation tasks (e.g., Vance et al., 2004; Marchant et al., 2008, 2009), which will be discussed later. Collectively, these findings are indicative of reflexive automatic control processes when an external focus is adopted. Importantly, the observed mechanisms suggest that accuracy or outputs are improved in line with more efficient movements when attention is directed to an intended outcome.

The constrained action hypothesis (Wulf et al., 2001a) proposes that internally focused instructions encourage conscious control which constrains the motor system through greater attentional demands and "noise" in the motor system. An external focus promotes automatic control processes which underpin effective and efficient movement execution. Supporting this, Castaneda and Gray (2007) demonstrated that attention is required to be directed externally toward a movement outcome (rather than simply distracted from movement awareness) as this supports the link between action effect representations and the automatic motor codes underpinning those effects (e.g., common-coding theory, Prinz, 1990).

Why should we be interested in instructions during force production? Given the consistent evidence on the benefits of externally focused instructions, one might suggest that individuals therefore naturally utilize this phenomenon when (a) learning movements themselves and (b) when instructing others. Neither seems to be the case. Firstly, evidence suggests that when not provided with instructions, participants appear to direct attention internally (e.g., McNevin and Wulf, 2002; Landers et al., 2005). Secondly, although the evidence is limited, professionals have been shown to deliver more internal than external instructions in movement settings (e.g., Durham et al., 2009). Coupled with researchers suggesting a lack 
of appreciation of attentional factors in strength training settings (Ives and Shelley, 2003), research assessing, and promoting effective instruction in force generation settings is necessary. Efficient and accurate force production activities require effective intra- and inter-muscular coordination, the magnitude of which Sahaly et al. (2001) have suggested could be sensitive to instruction. Researchers have recently attempted to address the influence of attentional focusing instructions on such variables, but one could argue that this interest is not entirely new. One of the first studies concerning the influence of attentional focusing instructions on motor control had its roots in the control of forceful action. Wulf et al. (1998) demonstrated that externally focused instructions on force production (onto the wheels of the apparatus) during a ski-slalom simulation task were beneficial to novices, acquisition, and retention of the skill compared to internally focused instructions (onto their exerting force with their feet) and a control condition. Subsequently however, little research has adequately addressed how instruction directs attention and influences performance in strength testing and training, despite verbal instruction and encouragement to increase muscular output being common practice (Campenella et al., 2000). Indeed, the details of instructions and encouragement and how they direct attention are rarely provided in research, despite Tod et al. (2003) highlighting attention as a key mechanism associated with improved performances in such tasks.

This review will attempt to draw together specific and related research addressing the influence of attentional focusing instructions on force production. To reflect the nature of the tasks employed in the research to date, the review will focus upon maximum force production (tasks assessing participants" ability to produce maximum force under different conditions); accurate force production (tasks aimed at assessing participants" ability to produce specific force levels); and prolonged force production (tasks requiring the continuous production of force over set distances or until failure due to fatigue). We also discuss research which attempts to influence force production through instruction, but not with the specific rationale of manipulating attentional focus. Viewing such research from the perspective of attentional focus manipulations allows for further insights to be drawn upon the nature and influence of instructions in these settings. Finally, the review draws together these different areas to allow for a discussion of current knowledge, implications and future directions. Table 1 details the instructions used in those studies which have specifically aimed to influence force production through verbal attentional focusing instructions.

\section{MAXIMUM FORCE PRODUCTION}

The ability to generate maximal forces is critical to many sporting, rehabilitative, exercise, physical therapy, and physical testing settings and procedures. In all such settings, researchers, and practitioners will instruct participants to guide effective movement execution. However, what has not been clear is the influence these instructions may have on participants' attentional focus and subsequent performance. Utilizing the vertical jump-and-reach task to assess maximal force production from whole-body coordination, Wulf and colleagues have attempted to address the potential influences of attentional focusing instructions on maximum force production. In the initial study, Wulf et al. (2007) directed attention either onto the rungs (of the Vertec measurement device) being reached for (external focus) or onto the fingers reaching for this rung (internal focus). In Experiment 1, an external focus resulted in significantly greater jump-and-reach height when compared to an internal focus and under control (no attentional focusing instruction provided) conditions in a within subject design. Given that within air movement patterns (e.g., differences in the reaching movement, not actual jump height) may have caused such an effect, in Experiment 2, center-of-mass (COM) displacement was also measured. Participants greater jump height using external instructions was associated with greater COM displacement compared to when internally focused instructions were utilized. This study therefore presented some of the first direct evidence that verbal external attentional focus manipulation benefits maximal force production as increased jump height was associated with increased force production. Supporting other research, an internal focus was shown to not differ from the no-instruction condition, suggesting that in force production tasks attention could be drawn internally without specific direction.

Replicating this jump-and-reach benefits of an external focus Wulf and Dufek (2009) and Wulf et al. (2010) also assessed a number of kinematic and neuromuscular variables (e.g., lower-extremity joint moments). In these studies, internally focused instructions (focusing on the fingers reaching for the rung of the apparatus) resulted in lower jump-and-reach height, COM and jump impulse displacement in a within-subjects design compared to externally focused instructions (focusing onto the rung being reached for). Wulf and Dufek also demonstrated that lower-extremity joint moments of the ankle, knee, and hip joints were significantly larger when externally focused instructions were provided compared to internal focus instructions, indicating benefited jump kinematic coordination. Whilst, Wulf, Dufek, Lozano, and Pettigrew attempted to explain the benefits in terms of activity and coordination patterns among associated muscles (tibialis anterior, biceps femoris, vastus lateralis, rectus femoris, lateral gastrocnemius), no differences in the pre-take-off muscle onset suggested that attentional focus did not influence coordination among muscle groups. The increased jump-and-reach height associated with externally focused instructions was achieved with lower EMG activity when compared to the internal instructions, suggesting that coordination within muscles is benefited through an external focus.

Collectively, these studies (Wulf et al., 2007, 2010; Wulf and Dufek, 2009) demonstrated that external attentional focusing instructions onto the movement outcome (object reached for) resulted in greater jump-and-reach heights compared to internally focused instructions (hand reaching with). This increased jump height was achieved through greater force production, which itself was the result of improved lower-extremity joint movements (Wulf and Dufek, 2009) and enhanced neuromuscular coordination (Wulf et al., 2010). Note that the instructions only manipulated attention around the reaching movement (e.g., targeted rung, or reaching finger). In balance studies utilizing supra-postural tasks (e.g., standing balance whilst touching and keeping a flexible curtain still, or dynamic balance whilst holding a bar level) where attention is manipulated internally and externally toward those tasks, whole-body performance is influenced (e.g., McNevin and Wulf, 2002; Wulf et al., 2003). Similarly, attentional focusing instructions have been shown to influence activity in muscles that are not 
specifically the focus of the attentional instructions (e.g., Vance et al., 2004; Zachry et al., 2005). Such influences have important implications for manipulating attentional focus in other force generating movements that require whole-body coordination toward a single output.

Recently, Porter et al. (2010) demonstrated that instructing attentional focus externally enhances standing long-jump performance compared to internally focused instructions. External instructions in this case directed attention toward "jumping as far past the start line as possible" whilst internally focused instructions emphasized "extending your knees as rapidly as possible." Although this study did not address kinematic or force production directly, the results add to those already discussed in highlighting the influence of different instructions on force production requiring whole-body coordination. However, of further interest is the effect of attentional focusing instructions upon force production that requires the manipulation of a specific object. Such tasks reflect many occupation, sporting and exercise tasks requirements, and are reviewed next.

The initial aim of Vance et al. (2004) was to compare muscular activity (integrated electromyography, iEMG) between different attentional conditions. In two experiments, experienced exercisers lifted a weighted barbell ( $50 \%$ of their maximal force production) using a biceps curl movement in a standing position. Attentional focusing instructions emphasized either the movement of the curl bar (external focus) or the arm and muscular movements (internal focus). In these experiments, iEMG of both the agonist (biceps) and antagonist (triceps) was significantly lower with externally compared to internally focused instructions. This provided initial evidence of more effective neuromuscular coordination induced through external instruction. Additionally, in the first experiment, externally focused instructions were associated with faster movement times compared to internal instruction. The second experiment controlled average movement speed using a metronome. As the weight lifted in each condition was the same, these findings led Vance et al. to suggest potential benefits of externally focused instructions when the goal of a task is force production due to the more efficient muscular activation patterns observed. Specifically, "focusing on the object that the force is being exerted upon may result in more effective performance than would focusing upon the body movements that produce the action" (p. 458). Research in our labs (Marchant et al., 2008) further replicated this muscular activity finding with further control over the movement timing. In that study, experienced exercisers completed elbow flexions at $60^{\circ} \mathrm{s}^{-1}$ on a isokinetic dynamometer under internal and external instructional conditions. Again, externally focused instructions were associated with lower biceps EMG when compared to internal and control instructions.

Following up these findings, we (Marchant et al., 2009) attempted to directly address the proposal that externally focused instructions could benefit maximal force production. Using an isokinetic dynamometer, experienced exercisers completed elbow flexions at $60^{\circ} \mathrm{s}^{-1}$ with the aim of producing maximal force during the full range of each movement. Instructions focused on the movement of the bar of the crank arm (external) or the movements of their arms and muscles (internal) during each lift. Significantly greater net joint torque (peak and integrated elbow flexor torque) and lower levels of muscular activity of the biceps (peak and integrated EMG) were observed when attention was directed externally compared with internally. As such, maximal voluntary force production was benefited from an external focus onto the object force is being exerted against as proposed by Vance et al. (2004). Focusing internally has resulted in elevated muscular activity that has not transferred to the movement output. However, no measurement of the antagonist muscle in this study means that discussion of intramuscular coordination is not possible. Efficient force production requires effective recruitment and coordination of fibers within and between agonist and antagonist muscles (e.g., Wulf et al., 2007). As we will see later, evidence suggests that attentional focus influences antagonist activation during force production. Despite the benefits to force production, participants in Marchant et al.'s study expressed a preference for internal over external instructions and rated the latter as harder to follow. However, no manipulation check in this study means that information on instruction use was lacking.

\section{ACCURATE FORCE PRODUCTION}

Those studies presented so far provide evidence that when instructions direct attention externally, maximal forces can be produced more effectively. These external focus effects are associated with more effective muscular activation and movement patterns. However, one could argue that such tasks do not represent the subtleties sometimes required in force production settings. Sporting, exercise, and rehabilitative tasks often require the ability to repeatedly and accurately produce sub-maximal force levels. In a recent series of studies, Keith Lohse assessed the role of attentional focusing instructions on participants" ability to accurately generate targeted submaximal forces. Participants using external instructions (focusing on pushing against the force platform during an isometric plantar flexion task) were more accurate in producing the force and reduced movement preparation time compared to internal instructions (focusing on the calf muscles) when attempting to generate $25 \%$ of their maximum force for $4 \mathrm{~s}$, after previously training without attentional focusing instructions (Liao and Masters, 2001, Experiment 1). In Experiment 2, the benefits of externally focused instructions were greater for a lower target (25\% maximum force) compared to a higher target (50\% maximum force) force. Therefore, the benefits of an external focus of attention may increase with increasingly precise force production requirements (Liao and Masters, 2001). Externally focused training instructions facilitated retention and transfer testing performance when no instructions were provided, during which participants" self-reported attentional focus was clearly linked to the instructions they trained with. Such durability of instructional sets received during practice impacts upon subsequent unguided performance.

Lohse et al. (2010b) assessed neuromuscular correlates during their target-force production task. After training without attentional instruction, participants attempted to generate $30 \%$ of the maximum force for $4 \mathrm{~s}$ whilst using either internally (focusing on the calf muscles, specifically the soleus muscle) or externally (pushing against force platform) focused instructions. Supporting the findings of Liao and Masters (2001), internally focused instructions resulted in greater error in producing this target force. In addition, internally focused instruction increased cocontraction of the agonist (soleus) and antagonist (tibialis anterior), and increased 
recruitment of the antagonist (indicative of poor intra- and inter-muscular coordination (Lohse et al., 2010b). This latter finding supports research demonstrating increased muscular activity during internally focused instructed movement execution in sporting settings (e.g., Zachry et al., 2005). Related research supports the assertion that attentional focusing instructions influence the production of specifically targeted sub-maximal forces. Freedman et al. (2007), examined tongue and hand strengths in relation to attentional focusing instructions to assess their potential utility in speech therapy. Healthy undergraduate students produced rapid pressure bursts ( 40 bursts of $20 \%$ of their maximal strength, at $5 \mathrm{~s}$ intervals) of either hand or tongue during impulse force control tasks (suggested to result in relatively fatigue resistant muscular activation). Externally focused instructions (onto pressure exerted on an air-filled rubber bulb held in the mouth or hand) resulted in greater accuracy and less variability in the production of the target submaximal force level in both force control tasks when compared to internal instructions (onto exerting force with the hand/tongue).

In summary, the recent work of Liao and Masters (2001), Lohse et al. (2010b), and Freedman et al. (2007) is supportive of the work presented so far in that verbal instructions emphasizing internally referenced information (e.g., muscles and movements associated with force production) result in reduced muscular efficiency compared to externally focused instructions (e.g., the object force is being exerted against) during force production. Verbal instructions emphasizing attentional focus onto a force-plate (Liao and Masters, 2001; Lohse et al., 2010b), or a air filled pressure sensitive bulb (Freedman et al., 2007) being squeezed benefited the generation of force. However in these studies, the benefits have been demonstrated in relation to subtle and targeted sub-maximal rather than maximal force production tasks. Clearly these studies represent a limited body of evidence so far, and further work is required on a variety of tasks. Within those studies described, testing protocols required participants to repeatedly generate targeted sub-maximal forces that are relatively resistant to fatigue effects. Of interest next is how the beneficial effects of externally focused instructions on force production and movement efficiency translate to prolonged force generation or repetitive execution of forceful movements that are vulnerable to fatigue effects.

\section{PROLONGED FORCE PRODUCTION AND ENDURANCE}

As well as assessing the influence of attentional focusing instructions on maximal and accurate force production, researchers have recently attempted to ascertain their influence on the maintenance of force generation in muscular endurance tasks. Wulf and Lewthwaite (2010) proposed that an external focus should be associated with more effective maintenance of sub-maximal force production, whereas an internal focus would limit muscular endurance through inefficient movement and muscular activation patterns. Only a limited number of studies have used the internal and external focus conceptualization discussed so far in relation to prolonged sub maximal exertion type tasks. However, a large body of research in the sport and exercise psychology literature does address the impact of attentional focus on endurance. Conceptually, this operationalization of attentional focus has differed from that discussed so far. It is not the scope of this review to assess this large body of research (for a discussion, see Lind et al., 2009), which has primarily been concerned with the attentional focusing strategies of association (focus on bodily sensations) and dissociation (actively "blocking out" painful physiological responses related to task effort). What will be discussed here are those studies that have recently attempted to assess the influence of attentional focusing instructions on prolonged submaximal force production.

Research we conducted in an exercise setting (Marchant et al., in press) recently demonstrated the impact of attentional focusing instructions on trained individuals" muscular endurance. Using three typical exercise movements (bench press and squat), experienced exercisers used internally (e.g., focusing on the movements of the limbs involved in the exercise) and externally (e.g., focusing on the movement of and exerting force against the bar being lifted) focused instructions whilst executing continuous repetitions of standardized weights to failure. In the first exercise participants completed a modified version of the YMCA bench press test (with males lifting $40 \mathrm{~kg}$ and females lifting $20 \mathrm{~kg}$ to failure) on a Smith Machine (ends of barbell attached to free running bearings on two vertical bars allowing for vertical movement only). Externally focused instructions resulted in significantly more repetitions executed before failure than under the internal instruction condition, but not the control condition. In the second exercise, participants completed more repetitions on a free bench press (barbell is free from restriction, executed on a standard bench and rack) at $75 \%$ of their one Repetition Maximum (1RM, maximum weight an individual is able to lift in a single repetition of an exercise). A significantly greater number of repetitions were completed using externally focused instructions when compared to both internal and control instructions. Similar findings were observed in the final exercise, where external instructions resulted in greater repetitions to failure when compared to both internal and control instructions when participants executed Free Squat (free barbell held behind the neck and across the upper back, lifted with the legs) lifts at $75 \%$ of their $1 \mathrm{RM}$.

These three progressively more complex weight lifting movements demonstrated increasing sensitivity to the impact of attentional focusing instruction. With movement restricted to the vertical plane in the Smith Machine bench press, the benefits of an external focus were significant but minimal. The benefits increased when executing movements on the free bench press and during the more complex free squat exercise (in terms of musculature involved and motor unit coordination). These findings demonstrate the influence of subtly different instructional emphasis on this trained population's ability to maintain force production before failure, an effect that increases as movement complexity develops. However, data on the movement kinematics and neuromuscular variables is lacking, and further discussion is limited. Such data would benefit an understanding of how movement form deteriorated during these lifts to failure. For example, research demonstrates that good form deteriorates with fatigue (e.g., Duffey and Challis, 2007), and methods of reducing this would benefit training outcomes and safety. Similarly, movement time was neither recorded nor controlled. Given that Vance et al. (2004) observed faster movement execution times when an external focus was employed, control, or measurement of movement speed would benefit future research. 
Finally, Marchant et al. (in press) employed only basic checks of compliance. This limits discussion of participants' experiences and specific use of the instructions provided.

Freudenheim et al. (in press) have assessed how attentional focusing instructions influence intermediate swimmers" performance of a prolonged performance task ( $16 \mathrm{~m}$ front crawl). In a novel approach to the instructional manipulation, and one reflecting the complex coordinative nature of swimming, in their first experiment instructions not only directed attention internally and externally, but also toward either the arm and leg components of the stroke. When using internal instructions, participants focused on "pulling your hands back" (arm stroke) or "pushing the instep down" (leg kick). In the external condition, participants were instructed to focus on "pushing the water back" (arm stroke) or "pushing the water down" (leg kick). All participants completed internal and external instruction trials, but these were specific to the stroke component group that they participated in (e.g., arm pull or leg kick group). Swimming times were faster when using external instructions, regardless of the stroke component emphasized. Experiment 2 only emphasized attentional instructions of the arm pull, and also demonstrated faster swimming times for an external focus. As such, performance benefited from attention being directed toward a force-related outcome (pushing the water down or back) compared with internally focused instructions onto movement mechanics.

The novel approach to the instructional manipulation of an external attentional focus in this study generates further discussion. When manipulating an external focus in tasks such as swimming where clear outcomes are limited, Freudenheim and colleagues have emphasized the exertion of force against the water. This is in line with Wulf's (2007) suggestion that instructions which emphasize pushing the water back during swimming may hold external properties (see p. 65). With reference to swimming breaststroke, Wulf also suggests that instructions should emphasize the production of a triangle with the arms (see p. 62). Researchers should foster links and discussion with coaching practitioners to highlight potentially useful sources of external emphasis possibly drawing upon analogies (e.g., Liao and Masters, 2001).

Despite such findings, key limitations are also worth considering. Information concerning arm stoke parameters (length and frequency) and the quality of leg movements (e.g., number/type of cycles), would have allowed discussion of whether velocity was affected by changes in stroke length or stroke frequency. The prolonged nature of the sprint task does differ from previous research and provides useful insight, but it requires maximal power production rather than specifically muscular endurance.

In summary, there is limited research addressing the maintenance of force production using instructionally manipulated attentional focuses. However, initial evidence suggests that an external focus provides some protection against the development of fatigue so that performance can be maintained when compared to internal focused instructions. Research has emphasized an external focus onto the object or substance which force is being exerted onto (a barbell or the water through which one is swimming) in comparison to internal focus onto the limbs or muscles utilized in the movement. Information on the direct mechanisms of these effects is lacking, particularly in the form of movement kinematics, kinetics, and physiological parameters. Given the improved performance, it can be suggested that an external focus promotes movement efficiency and coordination, but research is required to directly assess this.

\section{NON-ATTENTIONAL FOCUSING INSTRUCTION AND FORCE PRODUCTION RESEARCH}

Researchers in physical performance and therapy settings have long been interested in how to influence force production, and verbal instruction and encouragement are regularly utilized in testing protocols. Despite the full details of instructions not being consistently reported in much force production research, some researchers have assessed the influences of different types of instructions albeit not from an attentional perspective. For example, Cowling et al. (2003) assessed the influence of instruction on the control of force during landing movements. Instructions emphasizing increasing knee flexion induced lower landing forces than those emphasizing earlier recruitment of the hamstring during landing and under noinstructions (see also Prapavessis and McNair, 1999; McNair et al., 2000), suggesting a protective benefit during control of landing forces if attention is directed to form. Although both instructions are internally referenced in terms of the present discussion, they differ in terms of joint-movement or muscular emphasis. Participants were unable to selectively recruit their hamstring when instructed to do so and inadvertently altered their quadriceps muscle activation to the extent that it was less protective during landing. A finding similar to that of Lohse et al. (2010b) discussed above. Without a specific external instructional set a direct comparison is difficult, but these findings do support the previous work highlighting harmful effects of focusing internally onto muscular activation whilst controlling landing forces. These landing tasks are without an obvious movement outcome for external manipulation, but the emphasis of good movement form (rather than mechanics) appears to offer the best alternative at present. Whilst Castaneda and Gray (2007) and $\mathrm{McNevin}$ et al. (2003) suggest that different external attentional focuses have different effects, in these cases, different internally referenced instructions have different effects. McNair et al. (2000) demonstrated decreased landing forces when attention was directed externally toward the sound of landing. But these benefits were only observed against a control and an imagery condition, and were not different from those gained from the instruction condition. McNair et al. (2000) concluded that instruction guiding safe jump landing should direct attention to the sound of participants" landing in addition to concise lower limb kinematic instruction. Whether such mixed attentional focus instruction can be effective is unclear, but research should identify relevant externally focused instructions that could be employed in such force control settings.

Other research addressing attentional manipulations and prolonged movement execution have not fully addressed the conceptualization of attentional focus presented so far. For example, Schücker et al. (2009) demonstrated that enhanced running economy values (lower oxygen consumption, but not blood lactate or heart rate) were related to the adoption of an external focus onto the surroundings when compared to focusing on breathing or running movements during a 30 -min run at $75 \% V_{\text {O2max }}$. A focus upon breathing also resulted in a slower deeper breathing rate. Participants indicated that the external condition was the easiest, and that the strategies were used during the majority but not all of 
the runs. Schücker et al. concluded that running (and associated breathing patterns) was at its most economic when the automatic control processes involved in its execution were not interfered with through conscious control. However, rather than an externally distracting comparison, an effective task-relevant external instructional set is required. Research identifying such information could potentially test Wulf's (2007) proposal that focusing upon stride length may be a useful external reference (p. 64, see also research on rehab). However, contrary to this Clingman and Hilliard (1990) demonstrated that focusing on cadence was superior to focusing on stride length and a dissociating condition in improving racewalkers performance. Research assessing the impact of different external instructions is warranted for tasks requiring prolonged force production. As already stated, different types or distances of external focus may have differential influences on force production, and this may be an important variable in endurance settings.

In reviewing these examples of forceful movement execution research it is clear that the identification and examination of different types of externally focused instruction is necessary for further analysis of tasks such as these. It is also apparent that more crossdisciplinary collaboration is required to disseminate the attentional focusing instruction research findings from movement science settings to strength and conditioning research and testing settings. Given the clear interest by a number of researchers in the influence of instruction on force production, such collaboration may provide a fruitful and insightful opportunity. Overall, it is clear that the emphasis of instructions provided has an impact on performance in the force production tasks discussed. It also appears quite likely that in each of the examples provided, the attention focus emphasized in the instructions provided is a key mechanism in the observed outcomes.

\section{DISCUSSION}

The available research findings presented in this review indicate that an external focus allows the motor system to "self-organize"; efficiently coordinating and directing forces needed for accurate, maximal and sustained force production. The observed associated mechanisms include improved limb coordination (e.g., Wulf and Dufek, 2009) and intra- and inter-muscular coordination (e.g., Marchant et al., 2009; Lohse et al., 2010b; Wulf et al., 2010). Together, these findings present the developing understanding of different attentional focusing instructions" influence on force production outcomes and mechanisms of performance. It appears so far that Vance et al.'s (2004) proposal that an external focus onto the object through which force is being exerted will be beneficial is correct. Clearly, the research here is at an early stage, and a number of issues require further consideration.

Given the nature of the force production tasks employed, different instructional approaches have been utilized (see Table 1). Studies have generally provided instruction prior to performance, but in some cases (often to control for encouragement) reminders and prompts are provided during tasks (e.g., Marchant et al., in press; Freedman et al., 2007). Similarly, the nature of the target-force production tasks employed by Liao and Masters (2001) required the provision of internally and externally focused feedback throughout trials. Research is required to assess the impact of such during task instruction and feedback, and given that encouragement during force production tasks has been shown to influence performance (e.g., Bickers, 1993; Campenella et al., 2000) some degree of control over this is required if the instructed attentional focus is to be consistent. Related to this, limited evidence on the use and experiences of the instructions provided was available. Over prolonged force production tasks, the issue of maintaining attentional focus becomes important in terms of general concordance and attentional capacity.

Whilst motor control researchers suggest a propensity to focus attention internally (primarily onto explicit awareness of skill execution) when under pressure and that this is a critical mechanisms in choking under pressure (Baumeister, 1984; Lewis and Linder, 1997; Beilock and Carr, 2001), researchers assessing exertional experiences and tolerances note that attention is drawn internally (primarily onto physiological feedback) under increasing workloads. The ability to shift between attentional focuses depends upon the intensity of exertion (see Tenenbaum and Hutchinson, 2007). For example, Hutchinson and Tenenbaum (2007) have demonstrated that at high intensity and prolonged duration workloads, attentional focus shifts internally and becomes less flexible as physiological sensations become increasingly salient. Whereas at lower and moderate levels of exertion, the individual can voluntarily shift attentional focus. This suggests a limit to the influence of instructional manipulations of attentional focus during force production tasks as workload increases. Can providing externally attentional focusing instruction and feedback facilitate efficient movements and force production during these difficult ranges of workload, or is an internal focus at these stages unavoidable?

The experiences of effort, force production and exertion are not adequately addressed in this body of research to date. For example, the feed-forward hypothesis proposes a relationship between neuromuscular activation and perceived exertion (e.g., Cafarelli, 1982; Hasson et al., 1989; Pincivero and Gear, 2000). As such, another limitation of an internal focus that it also increases perceptions of effort in line with associated increases in muscular activity? How instruction interacts with perceptions of effort in these settings is important information, and may be useful for guiding future instructional approaches. Similarly, no research has addressed how the effects of attentional focusing instructions in force production settings are moderated by participant characteristics such as expertise and experience, self-efficacy, or attentional control. The majority of the studies discussed have tested healthy undergraduate and relatively untrained populations. How well do the results discussed so far generalize to trained and experienced individuals?

Worth further consideration for the development of externally focused instructions, de Graaf et al. (2004) suggest that muscle force awareness is explicit knowledge of the muscular force produced during voluntary movement and is distinct from movement outcome awareness. In their study, de Graaf et al. demonstrate that whilst kinematic awareness is easily accessible, muscular force awareness is harder to perceive (both in terms of perceived demands and associated cortical activation). Given this difficulty, what implications are there for effective instruction of attentional focus during force production tasks? Reviewing the instructions used to date, for externally focused instructions some studies emphasize force production, others emphasize movement outcomes, and some mix both external components. Likewise, when manipulating an 
Table 1 | Attentional focusing instructions used in muscular force production research.

\begin{tabular}{|c|c|c|c|c|}
\hline \multirow[b]{2}{*}{ Force type } & \multicolumn{2}{|c|}{ Study details } & \multicolumn{2}{|c|}{ Attentional focusing instructions } \\
\hline & Reference & Task characteristics & Internal & External \\
\hline \multirow[t]{4}{*}{ Maximum } & $\begin{array}{l}\text { Vance et al. } \\
\text { (2004) }\end{array}$ & $\begin{array}{l}\text { Lifting a barbell in a biceps curl (50\% } \\
\text { of maximal force production). }\end{array}$ & $\begin{array}{l}\text { Concentrate on your biceps } \\
\text { muscles }\end{array}$ & Concentrate on the curl bar \\
\hline & $\begin{array}{l}\text { Wulf et al. (2007, } \\
\text { 2010), Wulf and } \\
\text { Dufek (2009) }\end{array}$ & Vertical jump-and-reach task & $\begin{array}{l}\text { Concentrate on the tips of your } \\
\text { fingers, reaching as high as } \\
\text { possible }\end{array}$ & $\begin{array}{l}\text { Concentrate on the rungs of the Vertec, } \\
\text { reaching as high as possible }\end{array}$ \\
\hline & $\begin{array}{l}\text { Porter et al. } \\
(2010)\end{array}$ & Standing long jump & $\begin{array}{l}\text { Focus your attention on extending } \\
\text { your knees as rapidly as possible }\end{array}$ & $\begin{array}{l}\text { Focus your attention on jumping as far } \\
\text { past the start line as possible }\end{array}$ \\
\hline & $\begin{array}{l}\text { Marchant et al. } \\
(2008,2009)\end{array}$ & $\begin{array}{l}\text { Maximal isokinetic elbow flexions on } \\
\text { a dynamometer }\end{array}$ & $\begin{array}{l}\text { Focus upon the movement of your } \\
\text { arm and muscles during the lift }\end{array}$ & $\begin{array}{l}\text { Focus upon the movement of the crank } \\
\text { handle during the lift }\end{array}$ \\
\hline \multirow[t]{3}{*}{ Prolonged } & $\begin{array}{l}\text { Marchant et al. } \\
\text { (in press) }\end{array}$ & $\begin{array}{l}\text { Repetitions to failure on: 1, restricted } \\
\text { bench press (males: } 40 \mathrm{~kg} \text {, females: } \\
20 \mathrm{~kg}) \text {; } 2 \text {, free bench press }(75 \% 1 \mathrm{rm}) \text {; } \\
3 \text {, free back squat ( } 75 \% 1 \mathrm{rm}) .\end{array}$ & $\begin{array}{l}\text { Focus on moving and exerting } \\
\text { force with your arms (bench press) } \\
\text { Focus on moving and exerting } \\
\text { force with your legs (Squat) }\end{array}$ & $\begin{array}{l}\text { Focus on moving and exerting force } \\
\text { through and against the barbell (all) }\end{array}$ \\
\hline & $\begin{array}{l}\text { Freudenheim } \\
\text { et al. (in press) }\end{array}$ & $16 \mathrm{~m}$ front crawl as fast as possible & Pull your hands back (arm) & Push the water back (arm) \\
\hline & & & Push your instep down (leg) & Push the water down (leg) \\
\hline \multirow[t]{4}{*}{ Accurate } & $\begin{array}{l}\text { Freedman et al. } \\
\text { (2007) }\end{array}$ & $\begin{array}{l}\text { Rapid pressure bursts of hand and } \\
\text { tongue impulse force control task } \\
\text { ( } 20 \% \text { of max strength) }\end{array}$ & $\begin{array}{l}\text { Keep focusing on your tongue/ } \\
\text { hand, focus on your tongue/hand. } \\
\text { Push with your tongue/hand. }\end{array}$ & $\begin{array}{l}\text { Keep focusing on the bulb, focus on the } \\
\text { bulb. Push on the bulb. }\end{array}$ \\
\hline & $\begin{array}{l}\text { Liao and } \\
\text { Masters (2001) } \\
\text { and Lohse et al. } \\
\text { (2010b) }\end{array}$ & $\begin{array}{l}\text { Isometric plantar flexion task: } 30 \% \text { of } \\
\text { maximum force (Lohse et al., 2010b) } \\
\text { and } 25 \text { and } 50 \% \text { of maximum force } \\
\text { (Liao and Masters, 2001) }\end{array}$ & $\begin{array}{l}\text { Initial instruction: focus on the } \\
\text { push of the muscle of the calf } \\
\text { against the platform, because this } \\
\text { is the muscle producing the force }\end{array}$ & $\begin{array}{l}\text { Initial instruction: mentally focus on the } \\
\text { push of your foot against the platform, } \\
\text { because the platform is recording the } \\
\text { force that you produce in this }\end{array}$ \\
\hline & & & in this experiment. Feedback: you & experiment. Feedback: you were under/ \\
\hline & & & $\begin{array}{l}\text { were under/over, focus on contract } \\
\text { the muscle harder/less. }\end{array}$ & $\begin{array}{l}\text { over, focus on pushing harder against } \\
\text { the platform harder/less }\end{array}$ \\
\hline
\end{tabular}

internal focus, some studies emphasize movement kinematics whilst some emphases muscular activation, and some attempt both (see Table 1). Research considering these issues will aid effective instruction development.

Other methodological consideration include sound measurement of the mechanisms associated with performance changes as recent work has not provided a clear picture of how different attentional instructions have changed movement execution. For example, no data on lifting kinematics or swimming stroke components is provided in Marchant et al. (in press) or Freudenheim et al. (in press) to explain the benefits of an external focus. Longitudinal research assessing the implications of the present findings on physiological adaptation processes resulting from training with different attentional strategies will also benefit this area. Ives and Shelley (2003) indicate an appropriate attentional focus is a key variable in developing specific physiological training adaptations, without which adaptations are limited. Evidence of the long term impact of attentional focusing instructions and feedback is currently lacking.

Finally, in addition to muscular activation and biomechanical mechanisms, recent evidence demonstrates influences of attentional focusing instructions at a motor-relevant cortical level. Greater activation of the primary somatosensory, motor, and insular cortices were observed when externally compared to internally focused instructions were utilized during the acquisition of a simple motor skill (key press sequence; Zentgraf et al., 2009). Whilst an external focus enhanced task-relevant tactile information processing necessary for the effective execution of environmental-outcome movements, internally focused instructions disrupted the efficient neural flow between sensory and motor areas. That attentional focusing instructions should have a similar influence during force production task seems plausible considering that evidence points to central neural commands being vital in such tasks (e.g., Gandevia, 2001) and that an inadequate activation of motoneurons required for effective force production is associated with suboptimal descending drive from the motor cortex (Taylor et al., 2000). For example, loss of force through fatigue occurs due to suboptimal output from the motor cortex. This is evident in the observed increases in force evoked through activation of the motor cortex by transcranial magnetic stimulation (TMS; e.g., Gandevia et al., 1996; Taylor et al., 2000). Gandevia (2001) recommended better instruction and feedback to minimize the restrictive impact of these "supraspinal" variables in testing and training settings, and we have seen here that the influence of instructions depends upon their emphasized direction of attentional focus. As such, attentional focusing instructions may be a practically accessible way of researchers and practitioners 
maximizing participants" output. Research is required to address the impact of attentional focusing instructions on different brain areas in line with observed force production, muscular activation, and biomechanical parameters.

\section{CONCLUSIONS}

Specific research on force production and attentional focusing instructions to date is relatively limited, but adds to the body of evidence demonstrating beneficial effects of externally compared to internally focused instructions on movement skill performance and acquisition. The evidence suggests that an external focus onto the object through which or toward which force is being exerted is beneficial to force production, compared to an internal focus toward the movements and muscles associated with the force production. Specifically, individuals can produce greater forces, more accurate targeted forces, or maintain force production for longer when instructed to focus externally. However, firm conclusions cannot be reached until further research addresses a number of key issues (e.g., mechanisms of effects, moderating variables, instructional consistency). The findings do support Wulf's (2007) proposal that an external focus of attention should promote the body's natural propensity to conserve energy through "coherence between the outcome and the sensory consequences of that action" (p. 121). Underpinning the force production benefits discussed, research highlights movement efficiency as a key mechanism being promoted through an external focus of attention (e.g.,

\section{REFERENCES}

Al-Abood, S.A., Bennett, S. J., Hernandez, F. M., Ashford, D., and Davids, K. (2002). Effects of verbal instructions and image size on visual search strategies in basketball free throw shooting. J. Sports Sci. 20, 271-278.

Baumeister, R. F. (1984). Choking under pressure: self-consciousness and paradoxical effects if incentives on skillful performance. J. Pers. Soc. Psychol. 46, 610-620.

Beilock, S. L., and Carr, T. H. (2001). On the fragility of skilled performance: what governs choking under pressure. J. Exp. Psychol. Gen. 130, 701-725.

Bell, J. J., and Hardy, J. (2009). Effects of attentional focus on skilled performance in golf. J. Appl. Sport Psychol. 21, 163-177.

Bickers, M. J. (1993). Does verbal encouragement work? The effect of verbal encouragement on a muscular endurance task. Clin. Rehabil. 7, 196-200.

Cafarelli, E. (1982). Peripheral contributions to the perception of effort. Med. Sci. Sports Exerc. 14, 382-389.

Campenella, B. J., Mattacola, C. G., and Kimura, I. F. (2000). Effect of visual feedback and verbal encouragement on concentric quadriceps and hamstrings peak torque of males and females. Isokinet. Exerc. Sci. 8, 1-6.

Castaneda, B., and Gray, R. (2007). Effects of focus of attention on baseball batting performance in players of differing skill levels. J. Sport Exerc. Psychol. 29, 60-77.

Clingman, J.M., and Hilliard, D. V. (1990). Race walkers quicken their pace by tuning in, not stepping out. Sport Psychol. 4, 25-32.

Cowling, E. J., Steele, J. R., and McNair, P. J. (2003). Effect of verbal instructions on muscle activity and risk of injury to the anterior cruciate ligament during landing. Br. J. Sports Med. 37, 126-130.

de Graaf, J. B., Gallaca, C., Pailhous, J. Anton, J.-L., Roth, M., and Bonnard, M. (2004). Awareness of muscular force during movement production: an fMRI study. Neuroimage 21, 1357-1367.

Duffey, M. J., and Challis, J. H. (2007). Fatigue effects on bar kinematics during the bench press. J. Strength Cond. Res. 21, 556-560.

Durham, K., Van Vliet, P. M., Badger, F., and Sackley, C. (2009). Use of information feedback and attentional focus of feedback in treating the person with a hemiplegic arm. Physiother. Res. Int. 14, 77-90.

Fasoli, S.E., Trombly, C.A., Tickle-Degen, L., and Verfaellie, M. H. (2002). Effect of instructions on functional reach in persons with and without cerebrov56, 380-390. ascular accident. Am. J. Occup. Ther.

muscular activity and limb coordination). However, research is required to substantiate these findings further, incorporating a greater appreciation of the variables associated with force production. The maximal and accurate production and maintenance of forceful movements requires a complex interaction between cognitive, psychological, and physiological variables. Of these many variables, attentional focus has been differentially defined and utilized in both research and practice. For a fuller understanding of the role of task-specific attentional focusing instructions on force production in the future, researchers should consider how those instructions interact with individual and task-specific characteristics.

Finally, practitioners and researchers involved in testing or training individuals in force production settings should be aware of the influence even subtle differences in the instruction and feedback they provide can have. Researchers in particular should provide details of the instructions and feedback they have used in force production and testing protocols. This would allow for better comparisons between studies and findings. Furthermore, control for instructional content within force production research protocols should be a priority. Any differences in the attentional focus emphasized in instructions and feedback provided may induce unplanned differences between and within conditions, participants, and trials. In terms of consistency in facilitating performance, such instructions should direct attention externally toward the intended outcome of the movement.

Freedman, S. E., Maas, E., Caligiuri, M. P., Wulf, G., and Robin, D. A. (2007). Internal vs. external: oral-motor performance as a function of attentional focus. J. Speech Lang. Hear. Res. 50, 131-136.

Freudenheim, A. M., Wulf, G., Madureira, F., Corrêa, S. C. P., and Corrêa, U.C. (in press). An external focus of attention results in greater swimming speed. Int. J. Sports Sci. Coach.

Gandevia, S. C. (2001). Spinal and supraspinal factors in human muscle fatigue. Physiol. Rev. 81, 1725-1789.

Gandevia, S. C., Allen, G. M., Butler, J. E., and Taylor, J. L. (1996). Supraspinal factors in human muscle fatigue: evidence for suboptimal output from the motor cortex. J. Physiol. 490, 529-536.

Hasson, S. M., Williams, J. H., and Signorile, J. F. (1989). Fatigue-induced changes in myoelectric signal characteristics and perceived exertion. Can. J. Sport Sci. 14, 99-102.

Hutchinson, J. C., and Tenenbaum, G. (2007). Attention focus during physical effort: the mediating role of task intensity. Psychol. Sport Exerc. 8, 233-245.

Ives, J. C., and Shelley, G. A. (2003) Psychophysics in functional strength and power training: review and implementation framework. J. Strength Cond. Res. 17, 177-186.
Landers, M., Wulf, G., Wallmann, H., and Guadagnoli, M.A. (2005). An external focus of attention attenuates balance impairment in Parkinson's disease. Physiotherapy 91, 152-185.

Lewis, B. P., and Linder, D. E. (1997). Thinking about choking? Attentional processes and paradoxical performance. Pers. Soc. Psychol. B 23, 937-944.

Liao, C. M., and Masters, R. S. W. (2001). Analogy learning: a means to implicit motor learning. J. Sports Sci. 19, 307-319.

Lind, E., Welch, A. S., and Ekkekakis, P. (2009). Do "mind over muscle" strategies work? Examining the effects of attentional association and dissociation on exertional, affective and physiological responses to exercise. Sports Med. 39, 743-764.

Lohse, K. R., Sherwood, D. E., and Healy, A. F. (2010a). How changing the focus of attention affects performance, kinematics, and electromyography in dart throwing. Hum. Mov. Sci. 29, 542-555.

Marchant, D., Greig, M., and Scott, C. (2009). Attentional focusing instructions influence force production and muscular activity during isokinetic elbow flexions. J. Strength Cond. Res. 23, 2358-2366.

Marchant, D. C., Clough, P. J., and Crawshaw, M. (2007). The effects 
of attentional focusing strategies on novice dart throwing performance and their task experiences. Int. J. Sport Exerc. Psychol. 5, 291-303.

Marchant, D. C., Clough, P. J., Crawshaw, M., and Andrew Levy, A. (2009). Novice motor skill performance and task experience is influenced by attentional focusing instructions and instruction preferences. Int. J. Sport Exerc. Psychol. 7, 488-502.

Marchant, D. C., Greig, M., Bullough, J., and Hitchen, D. (in press). Instructions to adopt an external focus enhance muscular endurance. Res. Q. Exerc. Sport.

Marchant, D. C, Greig, M., and Scott, C. (2008). Attentional focusing strategies influence bicep EMG during isokinetic biceps curls. Athl. Insights. Available from: http:// www.athleticinsight.com/Vol10Iss2/ MuscularActivity.htm. (Retrieved July 26, 2010).

McNair, P. J., Prapavessis, H., and Callender, K. (2000). Decreasing landing forces: effect of instruction. $\mathrm{Br}$. J. Sports Med. 34, 293-296.

McNevin, N. H., Shea, C.H., and Wulf, G. (2003). Increasing the distance of an external focus of attention enhances learning. Psychol. Res. 67, 22-29.

McNevin, N. H., and Wulf, G. (2002). Attentional focus on suprapostural tasks affects postural control. Hum. Mov. Sci. 21, 187-202.

Pincivero, D. M., and Gear, W. S. (2000). Neuromuscular activation and perceived exertion during a high intensity, steady state contraction to failure. Muscle Nerve 23, 514-520.

Porter, J. M., Ostrowski, E. J., Nolan, R. P., and Wu, W. F. W. (2010). Standing long-jump performance is enhanced when using an external focus of attention. J. Strength Cond. Res. 24, 1746-1750.

Prapavessis, H., and McNair, P. J. (1999). Effects of sensory and augmented feedback on ground reaction forces when landing from a jump. J. Orthop. Sports Phys. Ther. 29, 352-356.

Prinz, W. (1990). "A common coding approach to perception and action," in Relationships Between Perception and Action, eds O. Neumann and W. Prinz (Berlin: Springer-Verlag), 167-201.

Sahaly, R., Vandewalle, H., Driss, T., and Monod,H. (2001). Maximal voluntary force and rate of force development in humans-importance of instruction. Eur. J. Appl. Physiol. 85, 345-350.

Schücker, L., Hagemann, N., Strauß, B., and Völker, K. (2009). The effect of attentional focus on running economy. J. Sport Sci. 27, 1241-1248.

Taylor, J. L., Allen, G. M., Butler, J. E., and Gandevia, S. C. (2000). Supraspinal fatigue during intermittent maximal voluntary contractions of the human elbow flexors. J. Appl. Physiol. 89, 305-313.

Tenenbaum, G., and Hutchinson, J. C. (2007). "A social-cognitive perspective of perceived and sustained effort," in Handbook of Sport Psychology, 3rd Edn, eds G. Tenenbaum and R. C. Eklund (Chichester: John Wiley), 560-577.

Tod, D. A., Iredale, F. F., and Gill, N. (2003). "Psyching-up" and muscular force production. Sports Med. 33, 47-58.

Vance, J., Wulf, G., Töllner, T., McNevin, N., and Mercer, J. (2004). EMG activity as a function of the performer's focus of attention. J. Mot. Behav. 36, 450-459.

Wulf, G. (2007). Attention and Motor Skill Learning. Champaign, IL: Human Kinetics.

Wulf, G. (2008). Attentional focus effects in balance acrobats. Res. Q. Exerc. Sport 79, 319-325.

Wulf, G., and Dufek, J. S. (2009). Increased jump height with an external attentional focus due to enhanced lower extremity joint kinetics. J. Mot. Behav. 41, 401-409.

Wulf, G., Dufek, J. S., Lozano, L., and Pettigrew, C. (2010). Increased jump height and reduced EMG activity with an external focus. Hum. Mov. Sci. 29, 440-448.

Wulf, G., Hö $\beta$, M., and Prinz, W. (1998) Instructions for motor learning: differential effects of internal versus external focus of attention. J. Mot Behav. 30, 169-179.

Wulf, G., Landers, M., Lewthwaite, R. and Töllner, T. (2009). External focus instructions reduce postural instability in individuals with Parkinson disease. Phys. Ther. 89, 162-168.

Wulf, G., Lauterbach, B., and Toole, T. (1999). Learning advantages of an external focus in golf. Res. Q. Exerc. Sport 70, 120-126.

Wulf, G., and Lewthwaite, R. (2010) "Effortless motor learning? An external focus of attention enhances movement effectiveness and efficiency," in Effortless Attention: A New Perspective in Attention and Action, ed. B. Bruya (Cambridge, MA: MIT Press), 75-101.

Wulf, G., McConnel, N., Gärtner, M. and Schwarz, A. (2002). Feedback and attentional focus: enhancing the learning of sport skills through external-focus feedback. J. Mot. Behav. 34, 171-182.

Wulf, G., and McNevin, N. H. (2003). Simply distracting learners is not enough: more evidence for the learning benefits of an external focus of attention. Eur. J. Sport Sci. 3, 1-13.

Wulf, G., McNevin, N. H., Fuchs, T., Ritter, F., and Toole, T. (2000). Attentional focus in complex motor skill learning. Res. Q. Exerc. Sport 71 229-239.

Wulf, G., McNevin, N. H., and Shea, C. H. (2001a). The automaticity of complex motor skill learning as a function of attentional focus. Q. J. Exp. Psychol. A. 54, 1143-1154.

Wulf, G., Shea, C. H., and Park, J. H. (2001b). Attention in motor skill learning: preferences for and advantages of an external focus. Res. Q. Exerc. Sport 72, 335-344.
Wulf, G., and Su, J. (2007). External focus of attention enhances golf shot accuracy in beginners and experts. Res. $Q$. Exerc. Sport 78, 384-389.

Wulf, G., Weigelt, M., Poulter, D. R., and McNevin, N. H. (2003). Attentional focus on supra-postural tasks affects balance learning. Q. J. Exp. Psychol. A 56, 1191-1211.

Wulf, G., Zachry, T., Granados, C., and Dufek, J. S. (2007). Increases in jumpand-reach height through an external focus of attention. Int. J. Sports Sci. Coach. 2, 275-284.

Zachry, T., Wulf, G, Mercer, J., and Bezodis, N. (2005). Increased movement accuracy and reduced EMG activity as a result of adopting an external focus of attention. Brain Res. Bull. 67, 304-309.

Zentgraf, K., Lorey, B., Bischoff, M., Zimmermann, K., Stark, R., and Munzert, J. (2009). Neural correlates of attentional focusing during finger movements: an fMRI study. J. Mot. Behav. 41, 535-541.

Conflict of Interest Statement: The author declares that the research was conducted in the absence of any commercial or financial relationships that could be construed as a potential conflict of interest.

Received: 26 July 2010; paper pending published: 14 September 2010; accepted: 04 November 2010; published online: 26 January 2011.

Citation: Marchant DC (2011) Attentional focusing instructions and force production. Front. Psychology 1:210. doi: 10.3389/ fpsyg.2010.00210

This article was submitted to Frontiers in Movement Science and Sport Psychology, a specialty of Frontiers in Psychology.

Copyright (c) 2011 Marchant. This is an open-access article subject to an exclusive license agreement between the authors and Frontiers Media SA, which permits unrestricted use, distribution, and reproduction in any medium, provided the original authors and source are credited. 